\title{
Summarization of Videos by Image Quality Assessment
}

\author{
Marcos Vinicius Mussel Cirne and Helio Pedrini \\ Institute of Computing, University of Campinas \\ 13083-852, Campinas, SP, Brazil
}

\begin{abstract}
Video summarization plays a key role in manipulating large amounts of digital videos, making it faster to analyze their contents and aiding in the tasks of browsing, indexing and retrieval. A straightforward method for producing the summaries is by means of extraction of color features from the video frames. However, in order to automatically generate summaries as human beings would do, the way that humans perceive images must be considered, which can be done by image quality assessment (IQA) metrics. This work presents VSQUAL, a method for summarization of videos based on objective IQA metrics, which is also used for other purposes such as shot boundary detection and keyframe extraction. Results of the proposed method are compared against other approaches of the literature with a specific evaluation metric.
\end{abstract}

Keywords: Video summarization, quality assessment, keyframe extraction.

\section{Introduction}

Recent advances in technology have made much easier for people to record digital videos. Everyday, they generate a huge amount of videos with their cameras and smartphones and, eventually, upload them on websites and social networks. Only on YouTube, an astonishing number of 100 hours of video is uploaded per minute [3]. Due to this fact, the demand for developing techniques which are capable of handling all these data in an automatic, efficient and accurate way increases drastically, in order to ease the tasks of searching, browsing, retrieving and content analysis. One of these techniques is video summarization, which generates a snippet from a given video, preserving as much relevant information as possible, such that the users can seize the central message transmitted by the original video. The generated summaries can then be integrated into many applications, such as interactive searching and browsing systems, making both management and access to video content more accurate [15, 18.

Nevertheless, defining what is important or not in video summarization is an open problem, especially because there is a variety of video genres, such as sports, movies, news programs, documentaries, and home movies in general. Even to humans, it is hard to reach a consensus to know how good a summary is, since what is relevant to ones may not be to others. Thus, the main challenge of the 
video summarization field is in how to make a system to take the best decisions to choose the most important parts of a video. This is usually done by analyzing high level features (e.g. semantic content, time, space) or low level features (e.g., color histograms, texture, subtitles, audio, shape and motion descriptors) 8].

A fundamental task in the process of video summarization is the selection of the features that best describe the video frames. Several approaches turn to color features (sometimes together with motion or spatio-temporal features), which requires a representation that is as close as possible to the way that humans perceive images. A popular approach is the use of HSV color histograms [5,7,11, 14, 16, but once they have many possibilities of construction (i.e., many choices for the number of bins for each band), the way that video frames are described varies from an approach to another.

Aside from the color histogram representation, another way to analyze images is by means of image quality assessment (IQA) metrics [13, 19, 20], which encompass physiological and psychophysical parameters related to the human visual system (HVS) at the same time that they take into account the structural information of the images. These metrics can be divided into two categories: subjective and objective. The former consists of selecting a number of users which evaluate the quality of a set of images and then a score is computed from this evaluation. However, this kind of method is time-consuming and inappropriate for real-time and automated applications. On the other hand, the latter focuses on automatic prediction of perceived image quality, where the scores for each image analysis are much faster to compute.

The objective of this work is to extend upon our previous video summarization method [8], and propose a new method called VSQUAL (Video Summarization by Image QUALity Assessment) using objective IQA metrics to evaluate video frames, together with new algorithms for some stages of the methodology pipeline. In addition, a more qualitative evaluation of the summaries is conducted, comparing the obtained results with a specific database.

The contributions of this work include the creation and implementation of a method that can be integrated into many video processing environments and a consistent analysis of the results, considering the variety of existing video genres.

This paper is organized as follows. Section 2 describes the main concepts and works related to video summarization and IQA. Section 3 presents the VSQUAL approach. Section 4 discusses some of the obtained results with VSQUAL. Section 5 includes the general conclusions about the discussed topic and some directions for future work.

\section{Background}

This section describes concepts about video summarization and IQA techniques.

\section{$2.1 \quad$ Video Summarization}

A hierarchical classification and overview of video summarization techniques is shown in [4, together with their respective advantages and drawbacks. These 
techniques were primarily divided into six categories, based on the mechanisms adopted in the summarization process. The categories include: feature [5], which analyzes low-level features such as color, texture, motion, etc.; clustering 9, which groups frames that have similar contents and generally used with clustering algorithms, such as $K$-means or some variation; event [12], that deals with the task of finding interesting events in a video; shot selection [10], which segments videos into shots and analyzes their degree of importance; trajectory [17, that focuses on analyzing dynamic content, detecting the behavior of moving objects in videos; and mosaic [6], which constructs a panoramic image from consecutive frames that contain an important content.

Naturally, choosing the most suitable approaches to a video summarization technique depends on the domain (genre) of videos. For example, sports videos can be summarized by combining specific features (usually color and motion), clustering and event approaches; in case of surveillance video summarization, a trajectory analysis may also be considered; movie highlights can be produced by adding shot selection and mosaic approaches, besides including audiovisual features, and so on. For a summarization technique which handles with several domains, the most common approaches are the ones that combine the first two categories, since the remaining ones make use of subjective elements (e.g. interesting events, degree of importance), which vary from one domain to other, or elements that rely on the dynamics of videos, i.e., trajectories (for videos with much dynamic content) and mosaic (more applicable to videos whose contents do not change rapidly) 4 .

In order to evaluate the effectiveness of a video summarization method, it is necessary a comparison against other approaches. However, since a consistent evaluation framework does not exist at the moment, each work has its own evaluation method [18. This happens due to many issues, such as the lack of an objective ground-truth and the difficulty in judging the quality of a summary (i.e., saying if a summary is better than another). Recently, some works have been using a common ground-truth together with a specific evaluation metric [5,7,14].

\subsection{Image Quality Assessment (IQA)}

In the last decades, a great amount of research has been made to develop objective image quality metrics that correlate with the way that humans perceive images, exploring some of the characteristics of the HVS. Most of these techniques are known as full reference (FR), which means that an original "distortion-free" image (reference) is compared to another one that may have some kind of distortion. Then, a score is computed from this comparison, measuring the quality of the second image according to the first one. Two conventional metrics are the MSE (Mean Square Error) and the PSNR (Peak Signal-to-Noise Ratio) [19], which are very simple to calculate, once they operate directly on the image intensities. However, it has been shown that they are not very suitable for describing the human perception of visual quality.

Another FR metric, called SSIM (Structural Similarity Index) [13, 19], considers the structural information of the image, in the sense that image pixels 
have strong dependencies among them, especially when they are very close in the spatial domain, and these dependencies carry important information about the structure of the objects present in the image. Based on that, SSIM models images in terms of three different components: loss of correlation, luminance distortion and contrast distortion. Thus, it provides higher fidelity results than those from MSE and PSNR.

Many alternatives for the SSIM metric (including extensions) have been proposed. One of them, proposed by Zhang et al. [20], analyzes the fact that the HVS understands an image based on its low-level features. Such metric is named FSIM (Feature Similarity Index) and it is computed from two complementary features: phase congruency (PC), which measures the significance of a local structure in the image, and gradient magnitude (GM), which stores information about image contrast. For two input images, the FSIM value between them ranges from 0 to 1, where 1 represents that both images are exactly the same. Moreover, FSIM has an extension that deals with color images, called $\mathrm{FSIM}_{C}$.

\section{Methodology}

The methodology of this work is an enhanced version of the one proposed in [8, which used a spectral clustering algorithm for summarizing videos of any genre. However, since the video frames were represented by features extracted from keypoints detected by image descriptors, it was difficult to find out a metric that precisely evaluates the difference between pairs of images based strictly on those features and keypoint matchings. Hence, the result analysis was bound to be more subjective. To overcome those problems, the FSIM $_{C}$, described in Section 2.2, was selected as the similarity measure and the CUS metric [7] was used to compare the summaries against other methods of the literature.

Figure 1 shows a flowchart of the VSQUAL method. Initially, it samples the video in a smaller amount of frames ( 5 frames per second) than the total number of frames so that it saves some computational time at the remaining stages. From these frames, the similarity matrix $S$ is computed, where for each pair of frames $i$ and $j, S(i, j)=\operatorname{FSIM}_{C}(i, j)$.

After that, at the shot detection stage, the number of video shots is estimated by analyzing the FSIM $_{C}$ 's between consecutive frames, which correspond to the set of all $S(i, i+1)$ elements such that $i=1,2, \ldots, N-1$, where $N$ is the total number of sampled frames. Here, intervals of higher values indicate that frames associated to each interval belong to the same shot, which happens because consecutive frames of a shot generally have similar contents and, therefore, high $\mathrm{FSIM}_{C}$ values. Moreover, whenever there is a significant decreasing in the $\mathrm{FSIM}_{C}$ values, it means that a shot transition may be detected, since the contents of a different shot are about to appear at a video. Then, the values become higher again, representing the frames of a new shot.

Hence, the shot boundary detection problem can be reduced to the problem of finding local minima points in a sequence of FSIM $_{C}$ between consecutive frames of a given video. To do this, a sliding window of size $m$ is used in order to look for large variations in the sequence. Starting from $i=1$, all points from $i$ to 


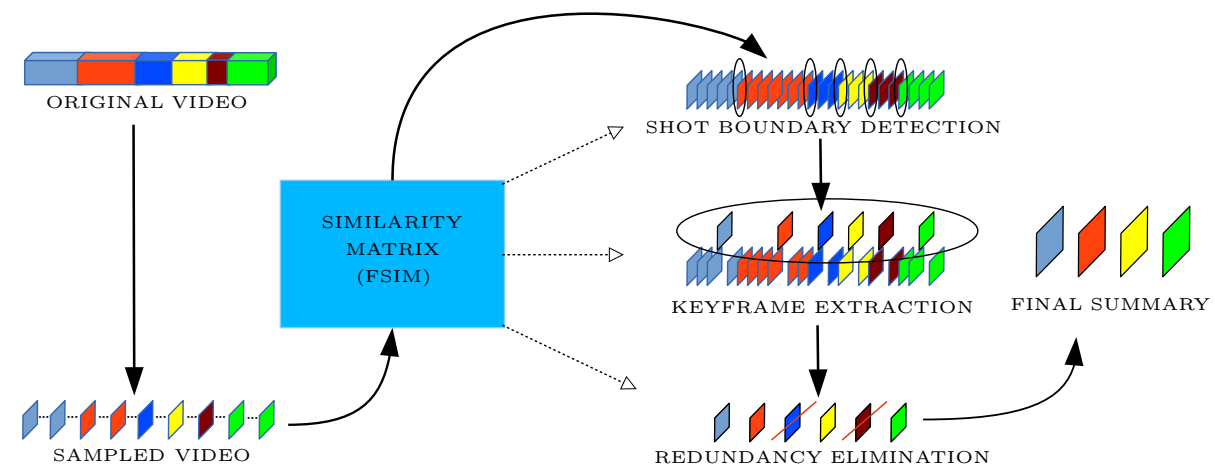

Fig. 1. Flowchart of the main stages of VSQUAL

$i+m-1$ are inserted into the window. Then, if the middle value is the lowest among all points and the difference between the maximum and the minimum values of the window is greater than a threshold $T_{1}$, the middle point will be regarded as a shot boundary. In this process, the values for $m$ and $T_{1}$ were 9 and 0.1 , respectively.

From the detected shot boundaries, the keyframe extraction process is then started, which selects one representative frame for each shot. Let $K$ be the total number of shots, which is the number of detected boundaries plus one. Also, let begin $_{k}$ and $e n d_{k}$ be, respectively, the indices of the beginning and the ending frames of the shot $k$, with $k=1,2, \ldots, K$. Taking advantage of the similarity matrix $S$ calculated at the second stage, the representative frame of shot $k$, denoted by $\mathrm{RF}_{k}$, is defined as:

$$
\mathrm{RF}_{k}=\left\{\text { begin }_{k} \leq f \leq \text { end }_{k} \mid \max \sum_{g=\text { begin }_{k}}^{\text {end }} S(f, g)^{2}\right\}
$$

In other words, Equation 1 selects the frame $f$ that is the most similar to the other ones of the same shot, which corresponds to the line of the similarity submatrix related to shot $k$ whose $\mathrm{FSIM}_{C}$ values are usually very high. This approach works very well for videos with none or little movement, where the FSIM $_{C}$ values between consecutive frames are generally close to 1 and do not oscillate too much inside each shot.

Once all the representative frames are obtained, a redundancy elimination algorithm is run to eliminate similar keyframes. From $S$, the FSIM $_{C}$ 's between all pairs of representative frames are observed, and if a value is equal to or greater than a similarity threshold $T_{S}$, a frame is discarded. For this process, $T_{S}$ was fixed at 0.75 . Then, the remaining frames will make part of the final summary.

At the last stage, the produced summary is compared against summaries of other methods of literature using the CUS metric, proposed by Avila et al. [7]. The authors evaluated their summaries by using their own features used to describe the video frames, which include selecting a specific number of bins of the hue band of the HSV color histogram. On the other hand, Mahmoud et al. [14] 
modified the CUS metric so that it could evaluate the summaries according to their descriptor (combination of color and texture features). Therefore, our evaluation will be based on the $\mathrm{FSIM}_{C}$ metric.

The advantage of VSQUAL resides on the fact that the video frames are represented by a more objective measure, which reflects the way that humans perceive images, and eliminates the use of parameter-dependent methods, such as choosing numbers of bins of color histograms. Furthermore, comparing to our previous approach [8], VSQUAL does not employ clustering algorithms, extracting keyframes in a simpler way, and it can be easily adapted to any other image quality metric. However, this new approach still has some problems in dealing with videos that have much movement, which causes oscillations at the FSIM $_{C}$ 's between consecutive frames, thus being harder to find boundaries (especially at gradual transitions) and more likely to select different keyframes from other approaches' summaries, reducing the CUS scores.

\section{Experimental Results}

The VSQUAL approach was tested with a collection of 50 videos of several genres from Open Video Project (OVP) [1], available at the VSUMM database [2] (provided by the authors of the approach described in [7]), were used in the tests, together with the respective summaries produced by different video summarization methods, which include DT [16], STIMO [11], VSCAN [14, as well as the OVP summaries and the ones provided by VSUMM.

A modified version of the CUS metric [7] is used to evaluate the results. In this metric, for each video, the automatic summaries of each method are compared to manual summaries produced by 5 different users (ground-truth). If a pair of frames (one from an automatic summary and other from a user summary) is considered similar, they are removed from the next iteration of CUS. The concept of frame similarity is the same used in the redundancy elimination step described in Section [3, where two frames are considered similar if the $\mathrm{FSIM}_{C}$ value between them is equal to or greater than $T_{S}=0.75$.

The evaluation is based on three different values: number of similar frames $S F_{i}$ (i.e., frames from automatic summary that matched to frames from user summaries), number of frames in the automatic summaries $A S_{i}$ and number of frames in the user summaries $U S_{i}$, where $i \in\{1,2,3,4,5\}$ represents a specific user. From these values, both precision $P_{i}$ and recall $R_{i}$ values can be obtained, with $P_{i}=S F_{i} / A S_{i}$ and $R_{i}=S F_{i} / U S_{i}$. Since there is a trade-off between precision and recall [5], the F-measure is used as the quality assessment metric for the automatic summaries. For each video, the F-measure is defined as:

$$
\text { F-measure }=\frac{\sum_{i=1}^{5} \frac{2 \times P_{i} \times R_{i}}{P_{i}+R_{i}}}{5}
$$

Table 1 shows the F-measures for the summaries of some videos from the database for each method. Rather than analyzing the entire database, only a 
subset of videos was chosen due to space limitation of the paper. From the table, it can be seen that VSQUAL performs better than most of the methods of the literature, also being comparable to recent approaches such as VSUMM and VSCAN. Despite the good results, the proposed method still needs some adjustments, once using only the $\mathrm{FSIM}_{C}$ values for detecting similarities leads to several false negatives, thus producing low F-measure values in some cases.

Table 1. F-measures of the summaries produced by each method and video

\begin{tabular}{lcccccc}
\hline Video & VSQUAL & DT & OVP & STIMO VSUMM VSCAN \\
\hline The Great Web of Water (Seg. 2) & 0.745 & 0.198 & 0.707 & 0.680 & $\mathbf{0 . 8 4 0}$ & 0.527 \\
A New Horizon (Seg. 4) & $\mathbf{0 . 4 1 1}$ & 0.195 & 0.122 & 0.181 & 0.335 & 0.340 \\
Senses And Sensitivity (Lect. 2) & 0.800 & 0.444 & 0.667 & 0.625 & $\mathbf{1 . 0 0 0}$ & 0.727 \\
Exotic Terrane (Seg. 3) & $\mathbf{0 . 6 9 1}$ & 0.449 & 0.521 & 0.370 & 0.580 & 0.624 \\
America's New Frontier (Seg. 1) & $\mathbf{0 . 6 2 2}$ & 0.413 & 0.476 & 0.301 & 0.572 & 0.599 \\
America's New Frontier (Seg. 10) & 0.414 & 0.268 & $\mathbf{0 . 5 2 6}$ & 0.443 & 0.470 & 0.499 \\
Oceanfloor Legacy (Seg. 2) & $\mathbf{0 . 6 5 3}$ & 0.470 & 0.599 & 0.365 & 0.644 & 0.636 \\
Hurricane Force (Seg. 3) & 0.615 & 0.545 & 0.548 & 0.366 & $\mathbf{0 . 6 9 2}$ & 0.570 \\
Drift Ice (Seg. 6) & $\mathbf{0 . 9 6 4}$ & $\mathbf{0 . 9 6 4}$ & 0.766 & 0.749 & $\mathbf{0 . 9 6 4}$ & 0.916 \\
Drift Ice (Seg. 7) & $\mathbf{0 . 8 5 6}$ & 0.713 & 0.763 & 0.616 & 0.814 & 0.846 \\
\hline
\end{tabular}

\section{Conclusions}

This paper described a video summarization method using objective IQA metrics, which are developed in such a way that is close to the way that humans perceive image qualities. $\mathrm{FSIM}_{C}$ was chosen to describe the video frames. The methodology pipeline was strictly based on the construction of the $\mathrm{FSIM}_{C}$ similarity matrix, from which it was possible to execute three different algorithms: shot boundary detection, keyframe extraction and redundant frame elimination. The final summary is created after those steps and compared to summaries produced by different video summarization approaches through the CUS metric.

Results showed that the proposed method is comparable to the state-of-theart, even though improvements are needed in order to increase the accuracy in the similarity detection process. These improvements include: combination of FSIM $_{C}$ with other feature(s), such as motion and spatio-temporal features to overcome the problem of dealing with contents with intense movement; a further analysis of the shot boundary detection step; and development of other algorithms for choosing the representative frame of each shot, with the goal of producing summaries that are as close as possible to the way that humans do.

Acknowledgements. The authors are grateful to FAPESP and CNPq for the financial support.

\section{References}

1. The Open Video Project (2014), http://www.open-video.org

2. VSUMM (Video SUMMarization) (2014), https://sites.google.com/site/vsummsite 
3. YouTube (2014), http://www.youtube.com/yt/press/statistics.html

4. Ajmal, M., Ashraf, M.H., Shakir, M., Abbas, Y., Shah, F.A.: Video Summarization: Techniques and Classification. In: Bolc, L., Tadeusiewicz, R., Chmielewski, L.J., Wojciechowski, K. (eds.) ICCVG 2012. LNCS, vol. 7594, pp. 1-13. Springer, Heidelberg (2012)

5. Almeida, J., Leite, N.J., Torres, R.D.S.: VISON: VIdeo Summarization for ONline Applications. Pattern Recognition Letters 33(4), 397-409 (2012)

6. Aner-Wolf, A., Kender, J.R.: Video Summaries and Cross-Referencing Through Mosaic-Based Representation. Computer Vision and Image Understanding 95(2), 201-237 (2004)

7. de Avila, S.E.F., Lopes, A.P.B., da Luz Jr., A., de Albuquerque Araújo, A.: VSUMM: A Mechanism Designed to Produce Static Video Summaries and a Novel Evaluation Method. Pattern Recognition Letters 32(1), 56-68 (2011)

8. Cirne, M.V.M., Pedrini, H.: A Video Summarization Method Based on Spectral Clustering. In: Ruiz-Shulcloper, J., Sanniti di Baja, G. (eds.) CIARP 2013, Part II. LNCS, vol. 8259, pp. 479-486. Springer, Heidelberg (2013)

9. Damnjanovic, U., Fernandez, V., Izquierdo, E., Martinez, J.M.: Event Detection and Clustering for Surveillance Video Summarization. In: 9th International Workshop on Image Analysis for Multimedia Interactive Services, pp. 63-66 (2008)

10. Evangelopoulos, G., Rapantzikos, K., Potamianos, A., Maragos, P., Zlatintsi, N., Avrithis, Y.S.: Movie Summarization Based on Audiovisual Saliency Detection. In: International Conference on Image Processing, pp. 2528-2531 (2008)

11. Furini, M., Geraci, F., Montangero, M., Pellegrini, M.: STIMO: STIll and MOving Video Storyboard For The Web Scenario. In: Multimedia Tools and Applications, vol. 46, pp. 47-69. Kluwer Academic Publishers, Hingham (2010)

12. Hong, R., Tang, J., Tan, H.K., Ngo, C.W., Yan, S., Chua, T.S.: Beyond Search: Event-driven Summarization for Web Videos. ACM Transactions on Multimedia Computing, Communications, and Applications (TOMCCAP) 7(4), 1-18 (2011)

13. Hore, A., Ziou, D.: Image Quality Metrics: PSNR vs. SSIM. In: 20th International Conference on Pattern Recognition, Washington, DC, USA, pp. 2366-2369 (2010)

14. Mahmoud, K.M., Ismail, M.A., Ghanem, N.M.: VSCAN: An Enhanced Video Summarization Using Density-Based Spatial Clustering. In: Petrosino, A. (ed.) ICIAP 2013, Part I. LNCS, vol. 8156, pp. 733-742. Springer, Heidelberg (2013)

15. Money, A.G., Agius, H.: Video Summarisation: A Conceptual Framework and Survey of the State of the Art. Journal of Visual Communication and Image Representation 19(2), 121-143 (2008)

16. Mundur, P., Rao, Y., Yesha, Y.: Keyframe-Based Video Summarization Using Delaunay Clustering. International Journal on Digital Libraries 6, 219-232 (2006)

17. Qiu, X., Jiang, S., Liu, H., Huang, Q., Cao, L.: Spatial-Temporal Attention Analysis for Home Video. In: IEEE International Multimedia and Expo, pp. 1517-1520 (2008)

18. Truong, B.T., Venkatesh, S.: Video Abstraction: A Systematic Review and Classification. ACM Transactions on Multimedia Computing, Communications and Applications 3(1) (February 2007)

19. Wang, Z., Bovik, A.C., Sheikh, H.R., Simoncelli, E.P.: Image Quality Assessment: From Error Visibility to Structural Similarity. IEEE Transactions on Image Processing 13(4), 600-612 (2004)

20. Zhang, L., Zhang, L., Mou, X., Zhang, D.: FSIM: A Feature Similarity Index for Image Quality Assessment. IEEE Transactions on Image Processing 20(8), 2378$2386(2011)$ 